2018-3

\title{
Total Hemispherical Apparent Radiative Properties of the Infinite V-groove with Specular Reflection
}

\author{
Rydge B. Mulford \\ Brigham Young University \\ Nathan S. Collins \\ Brigham Young University \\ Michael S. Farnsworth \\ Brigham Young University \\ Matthew R. Jones \\ Brigham Young University \\ Brian D. Iverson

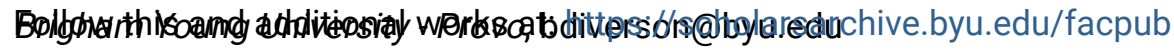 \\ Part of the Mechanical Engineering Commons
}

\section{Original Publication Citation}

Mulford, R. B., Collins, N. S., Farnsworth, M. S., Jones, M. R., and Iverson, B. D., 2018, "Total hemispherical apparent radiative properties of the infinite $\mathrm{V}$-groove with specular reflection," International Journal of Heat and Mass Transfer, Vol. 124, pp. 168-176.

\section{BYU ScholarsArchive Citation}

Mulford, Rydge B.; Collins, Nathan S.; Farnsworth, Michael S.; Jones, Matthew R.; and Iverson, Brian D., "Total Hemispherical Apparent Radiative Properties of the Infinite V-groove with Specular Reflection" (2018). Faculty Publications. 2158.

https://scholarsarchive.byu.edu/facpub/2158

This Peer-Reviewed Article is brought to you for free and open access by BYU ScholarsArchive. It has been accepted for inclusion in Faculty Publications by an authorized administrator of BYU ScholarsArchive. For more information, please contact ellen_amatangelo@byu.edu. 


\title{
Total Hemispherical Apparent Radiative Properties of the Infinite V-groove with Specular Reflection
}

\author{
Rydge B. Mulford, Nathan S. Collins, Michael S. Farnsworth, Matthew R. Jones, Brian D. Iverson ${ }^{1}$ \\ Department of Mechanical Engineering \\ Brigham Young University \\ Provo, UT, 84602
}

\section{ABSTRACT}

Multiple reflections in a cavity geometry augment the emission and absorption of the cavity opening relative to a flat surface in a phenomenon known as the cavity effect. The extent of the cavity effect is quantified using apparent absorptivity and apparent emissivity. Analysis of complicated thermal systems is simplified through application of apparent radiative properties to cavity geometries. The apparent radiative properties of a specularly-reflecting, gray, isothermal V-groove have been derived analytically, but these results have not been validated experimentally or numerically. Additionally, the model for apparent absorptivity of an infinite Vgroove subjected to partial illumination in the presence of collimated irradiation is not available. In this work, the following existing models for a specularly-reflecting V-groove are collected into a single source: (1) the apparent absorptivity of a diffusely irradiated V-groove, (2) the apparent emissivity of an isothermal V-groove and (3) the apparent absorptivity of a V-groove subject to collimated irradiation with full-illumination. Further, a new analytical model is developed to predict the apparent absorptivity of an infinite V-groove subject to collimated irradiation with partial-illumination. A custom, Monte Carlo ray tracing solver is used to predict the apparent radiative properties for all cases as a means of numerical verification by comparing the ray tracing data with the results from the new model in this work and the previously existing models. For diffuse irradiation, the analytical model and ray tracing data show excellent agreement with an average discrepancy of $4.4 \times 10^{-4}$, verifying the diffuse-irradiation analytical model. Similar

\footnotetext{
${ }^{1}$ Author to whom correspondence should be addressed: bdiverson@byu.edu
} 
agreement is found for collimated irradiation, where the full and partial illumination models indicate average discrepancies of $4.9 \times 10^{-4}$ and $4.6 \times 10^{-4}$ when compared with ray tracing data.

Keywords: V-groove, cavity effect, variable emissivity surface, angular surfaces, specular reflection 


\section{INTRODUCTION}

V-groove geometries are found in a variety of mechanical designs such as deployable mechanisms [1, 2], solar absorbers [3-5], solar cells [6], thermal radiators [7] and in the natural world. The analysis of radiative heat transfer within systems that contain V-groove geometries (or any cavity geometry) is complicated by the occurrence of multiple reflections within the Vgroove. The results of this study demonstrate apparent radiative properties may be used to accurately model the effects of multiple reflections in specularly reflecting V-grooves. The apparent radiative properties of diffusely reflecting V-grooves are addressed by Mulford et al. [8].

Cavity geometries emit and absorb more thermal radiation than an equivalent flat surface (equal in size to the cavity opening) with the same thermal radiation properties and temperature as the cavity walls [9]. The augmentation of emission and absorption, termed the cavity effect, is quantified with apparent emissivity and apparent absorptivity, respectively. Apparent emissivity is defined as the emission from a cavity opening divided by the emission from an equivalent black surface stretched across the cavity opening having the same temperature as the cavity walls [10]. Likewise, the apparent absorptivity is defined as the ratio of the incident radiation absorbed by the cavity to the total incident radiation on the cavity opening $[11,12]$.

The apparent radiative properties of a cavity are a function of the intrinsic radiative properties of the cavity walls, the type of reflection (specular or diffuse), the overall cavity shape and the cavity geometry [13]. The apparent absorptivity of a cavity is also dependent on the nature of the irradiation entering the cavity (diffuse or collimated) and the angle of incidence if the irradiation is collimated [14]. Conversely, the apparent emissivity of a cavity is strongly affected by the temperature of the cavity walls [15]. Likewise, the apparent emissivity of an isothermal cavity is equivalent to the apparent absorptivity of the same cavity subjected to diffuse irradiation, regardless of reflection type [16].

Apparent radiative behavior for a wide variety of cavity shapes subjected to various temperature profiles, irradiation conditions, and intrinsic surface properties are available in the literature. Several excellent reviews detail the different approaches used by various researchers to determine these apparent radiative properties and provide an overview of their results $[9,10$, 
13]. The majority of the studies performed on cavity geometries have focused on spherical [11, 17], cylindrical [18-20] and conical geometries [21] with a few papers examining V-groove or rectangular-groove geometries $[12,22]$. The closed, circular shapes often emphasized in the literature (i.e. cylinder or cone) have been investigated primarily because they are the most efficient shapes for approximating blackbody emission.

A new application of the cavity effect using origami surfaces comprised of angular cavities has revitalized the study of V-groove geometries [23]. Variations to cavity geometries cause the apparent radiative properties of the cavity to likewise change. By actively controlling the geometry of a cavity, the apparent radiative properties of the cavity may be controlled, allowing for adjustments to the apparent radiative properties in real time. Actuation of origami tessellations provide the mechanism by which cavity geometry is modified, creating a variable emissivity/absorptivity surface [23]. This application has created renewed interest in the apparent radiative properties of the V-groove shape, as this shape best approximates the cavities inherent to origami tessellations. Several apparent radiative property studies have examined the V-groove geometry, including works by Sparrow [12, 24, 25], Zipin [22, 26], Hollands [7] and Daws [27]. However, these models do not encompass the full range of possible cavity angles, intrinsic surface properties and collimation angles.

This work compiles the works of previous authors related to the V-groove geometry and presents a new model that addresses partial illumination in the case of collimated irradiation to provide a suite of equations that may be used to calculate the apparent emissivity or apparent absorptivity of a specularly-reflecting V-groove. The apparent absorptivity of a cavity for the full range of possible intrinsic surface properties, V-groove cavity angles and illumination conditions (diffuse or collimated) can be predicted with these models. The apparent emissivity expression will be limited to the case of an isothermal cavity. All models reported in this paper, whether derived in this work or developed by other authors, have been verified using Monte Carlo ray tracing. The case of a diffusely-reflecting V-groove cavity is considered separately [8].

\section{METHODOLOGY}

\subsection{Apparent Absorptivity for Diffuse Irradiation (Apparent Emissivity for Isothermal Cavity)}


The apparent emissivity for an isothermal, specularly-reflecting, infinite V-groove subject to diffuse irradiation has been determined analytically by Modest [28] based on the work of Sparrow [24, 25] and Hollands [7] resulting in Eq. 1. The maximum number of reflections $(n)$ experienced by a ray is equivalent to the integer portion of $\pi / \phi$, where the angle $\phi$ (as shown in Fig. 1a) is given in radians. Ohwada [16] has shown that the apparent emissivity of an isothermal cavity and apparent absorptivity of a diffusely-irradiated cavity are equivalent regardless of reflection mode. As such, Eq. 1 can be used to predict both apparent emissivity and apparent absorptivity for these conditions.

$$
\varepsilon_{a}=\alpha_{a}=\frac{\varepsilon}{\sin \left(\frac{\phi}{2}\right)}\left[1-\varepsilon \sum_{k=1}^{n} \rho^{k-1}\left(1-\sin \left(k \frac{\phi}{2}\right)\right)\right]
$$

An additional model that describes the apparent emissivity of an isothermal V-groove is available from Daws [27]. However, usage of the model relies on tabulated solutions that are only available for a limited number of cavity angles and intrinsic emissivities. Likewise, Sparrow has developed a method to calculate the radiative heat loss from a V-groove cavity [25], but an equation that gives the apparent emissivity is not derived. Finally, Sparrow has also derived a model for the apparent absorptivity of a diffusely-irradiated V-groove [12], which is equivalent to the isothermal apparent emissivity. However, Sparrow's model is limited to cavity angles that are given by the equation $\phi=\pi / n$ where $n$ is a positive integer. As such, Modest's equation, which has only been published as a textbook example question, has been selected for its simple implementation and general applicability. Eq. 1 has been verified with Monte Carlo ray tracing as will be shown in this work.

\subsection{Apparent Absorptivity for Collimated Irradiation}

The apparent absorptivity for a specularly reflecting infinite V-groove subject to collimated irradiation (at a given angle $\gamma$, Fig. 1b) must be separated into two scenarios, each with its own model. In the full illumination case $(\gamma \leq \phi / 2)$, incident rays completely illuminate the cavity, falling on both the left and right panels of the V-groove as shown in Fig. 1b. In the partial illumination case $(\gamma>\phi / 2)$, rays initially impact only a portion of one $\mathrm{V}$-groove panel and do not fall on the other panel as shown in Fig. 1c. Sparrow and Lin [12] developed a model that applies 
to the case of a fully illuminated V-groove which is summarized in Section 2.2.1 to provide context for the partial illumination model developed in this work (Section 2.3.1).

\subsubsection{Fully Illuminated V-Groove}

As shown in Fig. 2a, a sample ray with a given angle of incidence $\gamma$ (defined in the clockwise direction from the surface normal) intersects the left V-groove panel at location $X$ ' and exits the cavity after reflecting twice, leaving the cavity as close to the left V-groove panel as possible without intersection. Any ray that initially intersects the left V-groove panel at a location below $X^{\prime}$ will reflect one additional time before leaving the cavity; all rays that intersect above

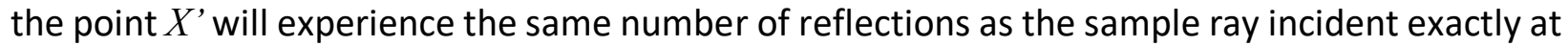
$X^{\prime}$. Fig. 2a also depicts the ray-plane angle $\beta$ (angle between an intersecting ray and the cavity surface) for both internal reflections and for the exiting ray. For the $i^{\text {th }}$ reflection, the ray-plane angle $\left(\beta_{i}\right)$ for a ray initially striking the left-hand panel is given by Eq. 2 [12]. The length $X$ ', which has been normalized by the length of the V-groove panel, is determined through the law of sines as given in Eq. 3 [12]. For the case of full illumination, the length $X^{\prime}$ is equivalent to $X_{n}$ or the fraction of rays incident on the left $\mathrm{V}$-groove panel that will reflect $n$ times.

$$
\begin{gathered}
\beta_{i}=\gamma+\left(i-\frac{1}{2}\right) \phi \\
X^{\prime}=\frac{\sin [(n-1 / 2) \phi+\gamma]}{\sin (\phi / 2+\gamma)}=X_{n}
\end{gathered}
$$

The maximum number of reflections experienced by rays initially incident on the left panel ( $n$ reflections for rays that intersect below $X^{\prime}$ ) is determined by assuming that a ray has left the cavity once the ray-plane angle of the next intersection $\left(\beta_{n+1}\right)$ exceeds $\pi$. When $\beta_{n+1}>\pi$, the ray is moving vertically upwards between the two V-groove planes such that an additional reflection is not possible. Setting $\beta_{i}=\pi$ in Eq. 2 and designating the $i^{\text {th }}$ reflection as $n$, the maximum number of reflections can be obtained as given in Eq. 4, where all angles are input as radians. The result of Eq. 4 must be rounded down to the nearest integer value, and if the initial evaluation is exactly an integer then the number of reflections should be decreased by one.

$$
n=\left\lfloor\frac{(\pi-\gamma)}{\phi}+\frac{1}{2}\right\rfloor
$$


Rays that initially strike the right surface also have a reflection split point $Y^{\prime}$, which is again equivalent to the fraction of rays that reflect $m$ times, or $Y_{m}$. The reflection split point and total number of reflections for the right side can be determined using Eqs. 3 and 4, respectively. However, in both equations $-\gamma$ should be used in the place of $\gamma$.

A percentage of the incident energy is absorbed $(\alpha)$ and reflected (1- $\alpha)$ at each reflection event. A summation of the total energy absorbed over all reflections (i.e. the total fraction of incident energy that is absorbed after $\mathrm{n}$ reflections) is given in Eq. 5, assuming that all of the incident energy reflected $n$ times.

$$
\alpha+\alpha(1-\alpha)+\alpha(1-\alpha)^{2}+\ldots+\alpha(1-\alpha)^{n-1}=1-(1-\alpha)^{n}
$$

In reality, only a fraction of the incident energy on one of the V-groove surfaces reflects $n$ times, that fraction being equivalent to $X_{n}$ or $Y_{m}$. To account for the unequal number of reflections, the right hand side of Eq. 5 is factored to give $1-(1-\alpha)(1-\alpha)^{n-1}$. The absorptivity of the factored term is then corrected by scaling the absorptivity by $X_{n}$ or $Y_{m}$ for the left and right sides, respectively, giving $1-\left(1-X_{n} \alpha\right)(1-\alpha)^{n-1}$ and $1-\left(1-Y_{m} \alpha\right)(1-\alpha)^{m-1}$. The corrected fraction of incident energy absorbed for each side of the V-groove is then multiplied by the incoming radiative flux and the projected area of the associated side. The sum total of both sides is divided by the product of the projected area of the $\mathrm{V}$-groove cavity and the incoming radiative flux. The radiative flux terms cancel in the numerator and denominator to give the following apparent absorptivity for a fully illuminated V-groove.

$$
\alpha_{a}=\frac{\left[1-\left(1-\alpha X_{n}\right)(1-\alpha)^{n-1}\right] \sin \left(\frac{\phi}{2}+\gamma\right)+\left[1-\left(1-\alpha Y_{m}\right)(1-\alpha)^{m-1}\right] \sin \left(\frac{\phi}{2}-\gamma\right)}{\left(2 \cos \gamma \sin \frac{\phi}{2}\right)}
$$

\subsubsection{Partially Illuminated V-Groove}

Sparrow and Lin [12] stated that an apparent absorptivity model for a partially illuminated V-groove with specular reflection would be similar to the model developed for full illumination (Section 2.2.1) but did not report this model or its development. This section outlines the development of a new model to determine the apparent absorptivity of a partially illuminated $\mathrm{V}$ - 
groove following the basic approach of Sparrow and Lin. The approach is also verified in this work with Monte Carlo ray tracing.

As with the case of full illumination, the total fraction of incident energy absorbed is determined through a summation, as shown in Eq. 5. The right hand side of Eq. 5 must again be scaled by $X_{n}$ to account for the uneven number of reflections, giving Eq. 7. The term for the right side of the $\mathrm{V}$-groove, or $1-\left(1-Y_{m} \alpha\right)(1-\alpha)^{m-1}$, is not present in the partial illumination model because no rays are initially incident on this side. The result given in Eq. 7 is the apparent absorptivity of a partially illuminated V-groove.

$$
\alpha_{a}=1-\left(1-\alpha X_{n}\right)(1-\alpha)^{n-1}
$$

For partial illumination, $X_{n}$ is not equivalent to $X^{\prime}$ due to shading of the left side of the $\mathrm{V}$ groove (Fig 1c). To find $X_{n}$ for a partially illuminated surface, it is first necessary to calculate the normalized length of the lowest ray's impact point ( $X^{\prime}$, Fig. 2b) using the law of sines, giving Eq. 8. The split point $X^{\prime}$ is calculated as before with Eq. 3. However, $X_{n}$ is now calculated using the following formulas for one of two scenarios: (1) if $X^{\prime}<X^{\prime}$, the split point $X^{\prime}$ falls below the lowest ray impact point $X$ ' ' indicating that all rays striking the left surface reflect a total of $n-1$ times and $X_{n}=0$; (2) if $X^{\prime} \geq X^{\prime}$, then $X^{\prime}$ must be scaled by $X^{\prime}$, as shown in Eq. 9, providing a value for $X_{n}$ that accounts for shading.

$$
\begin{gathered}
X^{\prime \prime}=\frac{\sin \left(\gamma-\frac{\phi}{2}\right)}{\sin \left(\pi-\frac{\phi}{2}-\gamma\right)} \\
\text { if } X^{\prime} \geq X^{\prime \prime}: X_{n}=\frac{X^{\prime}-X^{\prime \prime}}{1-X^{\prime}} \\
\text { if } X^{\prime}<X^{\prime \prime}: X_{n}=0
\end{gathered}
$$

The maximum number of reflections that an incident ray will experience before leaving the cavity $(n)$ must also be determined for a partially illuminated cavity. For the case of full illumination, a ray will continue to reflect inside of a cavity until the ray-plane angle (Eq. 2) exceeds $\pi$, allowing for $n$ to be found with Eq. 4. However, a ray reflecting inside of a partially illuminated $V$-groove could exit the cavity before the ray plane angle exceeds $\pi$. As an example, Fig. $2 b$ depicts a ray entering a partially illuminated cavity that is reflected twice before exiting. 
Upon exiting, it is clear that the ray-plane angle $\left(\beta_{3}\right.$ in Fig. $\left.2 b\right)$ of the exiting ray does not exceed $\pi$ but exits regardless due to the finite length of the $\mathrm{V}$-groove panels, violating the condition used in the derivation of Eq. 4.

Since Eq. 4 is not valid for partial illumination, the total number of reflections was determined through $2 \mathrm{D}$ ray tracing by tracking the path of a single ray that enters the cavity through the right-most point of the cavity opening (depicted in Fig. 2b) and counting the number of reflections the ray experiences before exiting the cavity. The mathematical development and final equations for the $2 \mathrm{D}$ ray tracing method are described in the Appendix. The value $n$ was obtained using the ray tracing routine detailed in the Appendix for all possible combinations of cavity angle $(\phi)$ from 0 to $\pi$ and ray incidence angle $(\gamma)$ from 0 to $\pi / 2$ in increments of $\pi / 180$ (i.e. $\left.1^{\circ}\right)$.

Fig. 3 illustrates the results for $n$ from the 2D ray tracing routine for all combinations of $\phi$ and $\gamma$ as a filled contour plot, where the dotted line illustrates the boundary between full and partial illumination. Distinct linear patterns, designated as solid black lines, are visible in both the full and partial illumination regions. In the full illumination region, the equation for each solid black line is given by $\gamma=-(n-1 / 2) \phi+\pi$, where $n$ is a positive integer and angles are in radians. When rearranged, this linear equation is equivalent to Sparrow's equation used to compute the number of reflections (Eq. 4) for full illumination. This agreement verifies Sparrow's counting method for the full illumination case.

As observed in Fig. 3 , the $n$-counting method used for full illumination is not applicable to the partial illumination case. However, a linear relationship between $\phi, \gamma$ and $n$ is also evident in the partial illumination region. Interpolation of the data following the same general form as Sparrow gives Eq. 10 which must also be rounded down to the nearest integer and angles are expressed in radians.

$$
n=\left\lfloor\frac{(\pi-2 \gamma)}{\phi}+1\right\rfloor
$$

For full illumination, Eq. 4 was derived with an intuitive physical argument. A similar physical argument for the derivation of Eq. 10 is less clear. However, if we rearrange Eq. 10, we 
obtain a form that is very similar to the fully illuminated case derived by Sparrow, indicating that half of the reflections have occurred once the ray-plane angle exceeds $\pi / 2$ (Eq. 11).

$$
\frac{n}{2}=\frac{(\pi / 2-\gamma)}{\phi}+\frac{1}{2}
$$

This interpretation is further verified by considering that a ray reflects downward as long as the ray plane angle is less than $\pi / 2$ and then reflects towards the direction of the opening once the ray plane angle has exceeded $\pi / 2$ (as indicated in Figs. $2 a$ and $2 b$ ), with the number of downward and upward reflections differing at most by a value of one.

\subsection{Monte Carlo Ray Tracing}

In the previous sections, analytical expressions have been reported or derived for the apparent absorptivity of an infinite V-groove and for the apparent emissivity of an isothermal Vgroove. Monte Carlo ray tracing was used to calculate the apparent absorptivity of the infinite Vgroove for equivalent heating conditions, providing a numerical verification of the analytical models. Ray tracing, a statistical approach, can be computationally expensive when compared with deterministic methods that incorporate specular reflection $[29,30]$. However, the simplicity of the application and the availability of computing power motivated the use of Monte Carlo ray tracing as a verification.

\subsubsection{Apparent Absorptivity}

Monte Carlo ray tracing is a straightforward numerical method used to solve radiation heat transfer problems with difficult geometries [10, 31, 32]. Emitted and absorbed rays for a geometry of interest are counted and related to apparent radiative properties. In this work, a direct relationship between the number of rays emitted and number of rays absorbed gives the apparent absorptivity of the tested cavity geometry.

A V-groove geometry was positioned directly below a transparent, emitting surface that provides a number of emitted rays downward into the $\mathrm{V}$-groove of cavity angle $\phi$. The rays may be emitted diffusely or at a given collimation angle $\gamma$ (see dashed lines in Figs. 1a - 1c). All rays emitted from the transparent surface enter the V-groove. The surfaces of the V-groove absorb a percentage of the incident rays equivalent to the intrinsic absorptivity of the material and reflect the remainder in a specular manner. Each ray is tracked until it is absorbed upon intersection 
with a cavity surface or escapes out of the cavity after one or more reflections. The total number of emitted rays $N_{T}$ and the number of absorbed rays $N_{a}$ are counted.

The apparent absorptivity of a surface is defined as the ratio of total absorbed energy $\left(q_{a}\right)$ to the total energy entering the cavity opening $\left(q_{t}\right)[12]$, as shown in Eq. 12. To determine apparent absorptivity from ray tracing results, each ray is assumed to represent a unit of quantized energy $[33,34]$. Each unit may be either completely reflected or completely absorbed at a single reflection event. With this analogy, the ratio of absorbed rays $\left(N_{a}\right)$ to the total number of rays $\left(N_{t}\right)$ can be used to obtain the apparent absorptivity as shown in Eq. 12 (see also [8]).

$$
\alpha_{a}=\frac{q_{a}}{q_{t}}=\frac{N_{a}}{N_{t}}
$$

In the case of diffuse irradiation, the apparent absorptivity of the infinite V-groove is equivalent to the apparent emissivity if the cavity is isothermal [16]. As such, ray tracing verification of Modest's equation (Eq. 1) was performed by determining the apparent absorptivity of the cavity and equating that value to the apparent emissivity as determined through Modest's model.

\subsubsection{Ray Tracing Application}

A custom ray tracing program was developed by the authors following the mathematical basis provided by Steinfeld $[8,11]$ to determine the values $N_{a}$ and $N_{t}$ for use in Eq. 12 . For diffuse emission, the polar and azimuthal angles of the emitted ray were determined through random number generation with the polar angle weighted towards a cosine distribution. Collimated rays were emitted at the specified collimation angle. The intersection of a ray and a participating surface were determined with a line-plane intersection algorithm [35]. When a ray-surface interaction occurred, a new random number in the range $[0,1)$ was compared against the absorptivity of the surface to determine if the ray was absorbed or reflected. If reflected, Equation 13 [11] was used to determine a new ray direction, where $\vec{r}_{2}$ is the reflected ray

direction vector, $\vec{r}_{1}$ is the incident ray direction vector and $\vec{v}$ is the unit normal vector of the impacted surface. This 3D ray tracing program was developed separately from the 2D ray tracing method described in the Appendix used for determining $n$ for the case of partial illumination. 


$$
\vec{r}_{2}=\vec{r}_{1}-2\left(\vec{v} \bullet \vec{r}_{1}\right) \vec{v}
$$

\subsubsection{Testing Procedure}

The custom ray tracing program was constructed in three-dimensional space for determining the apparent radiative properties of origami tessellations such as the accordion fold (Fig. 1d), the Barreto's Mars [2] and the Miura Ori [1]. To create the illusion of an infinite Vgroove, perfectly reflecting specular surfaces were added to the open ends of the 3D V-groove. A transparent surface was placed across the top opening of the V-groove of specified cavity angle and intrinsic emissivity. The transparent surface emitted a specified number of rays diffusely or in a collimated fashion into the cavity. The total number of rays emitted $N_{t}$ and number of rays absorbed $N_{a}$ was counted and the apparent absorptivity calculated using Eq. 12. Data was collected for all possible combinations of $\alpha, \phi$, and $\gamma$ (where applicable). The surface absorptivity $\alpha$ was varied from 0.01 to 1.00 in increments of $0.01 ; \phi$ from $\pi / 180$ to $\pi$ in increments of $\pi / 180$ $\left(1^{\circ}\right)$; and $\gamma$ from 0 to $8 \pi / 9$ in increments of $\pi / 18\left(10^{\circ}\right)$.

Each combination of $\phi, \gamma$, and $\alpha$ was evaluated a total of 20 times for $N_{t}$ number of rays. The standard error of the mean [36] for the 20 tests at a given value of $N_{t}$ was calculated using Eq. 14, where $S E$ is the standard error of the mean, $s$ is the unbiased standard deviation of the results and $N$ is the number of samples.

$$
S E=\frac{S}{\sqrt{N}}
$$

The number of total rays emitted was increased to $N_{t}=300,000$ rays, at which point the standard error of the mean fell at or below $5.0 \times 10^{-3}$ for all tested cases. A value of $5.0 \times 10^{-3}$ was determined to be an appropriate error threshold and all reported data was determined at this ray count.

\section{RESULTS AND DISCUSSION}

\subsection{Model Summary}

Table 1 provides the equation numbers, required inputs and references for the models developed by others and summarized in this work (i.e. for diffuse irradiation and for collimated irradiation with full illumination). The model for collimated irradiation with partial illumination was developed in this work and is also summarized in Table 1. Verification of these three models 
through Monte Carlo ray tracing is presented in Section 3.2 and the results of these models are presented in Section 3.3.

\subsection{Ray Tracing Verification}

\subsubsection{Apparent Absorptivity for Diffuse Irradiation (Apparent Emissivity for Isothermal Cavity)}

Fig. 4 depicts the results of the analytical model (Eq. 1 from Modest [28]) and ray tracing tests for a diffusely-irradiated absorbing infinite V-groove or isothermal, emitting V-groove. Results are compared for four different intrinsic surface properties. Although ray tracing was performed at 179 angles between $\phi=0$ and $\pi$, only a selection of the ray tracing data points are shown in the figure for clarity. The average discrepancy (the absolute value of the difference between the analytical result and ray tracing result) between the analytical model and all available ray tracing data is $4.4 \times 10^{-4}$ with a standard deviation of $9.8 \times 10^{-5}$. These values are within the average ray tracing error of $5.0 \times 10^{-3}$. As such, Eq. 1 is an accurate expression of apparent properties over the full range of intrinsic properties and cavity angle.

\subsubsection{Collimated Irradiation}

In Figs. $5 a$ and $5 b$, selected ray tracing results are provided to verify the results of the analytical models. The average discrepancy between all ray tracing data points for the fully illuminated region and analytical results is $4.9 \times 10^{-4}$ with a standard deviation of $7.8 \times 10^{-5}$. The average discrepancy is very small compared with the apparent absorptivity values, verifying Sparrow's model as accurate over the applicable range of cavity angles and collimation angles. In

the case of partial illumination, the average discrepancy between all ray tracing data points and analytical models is $4.6 \times 10^{-4}$ with a standard deviation of $8.1 \times 10^{-5}$. Again, these discrepancies are very small compared to the absolute apparent absorptivity values. This confirms the accuracy of the partial illumination analytical model introduced in this work.

\subsection{Apparent Radiative Properties}

\subsubsection{Apparent Absorptivity for Diffuse Irradiation (Apparent Emissivity for Isothermal Cavity)}

Fig. 4 illustrates the analytically derived apparent absorptivity for diffuse irradiation and the equivalent apparent emissivity for isothermal panels of an infinite V-groove as a function of cavity angle and intrinsic radiative property (Eq. 1). The influence of the cavity effect is clearly evident, where the apparent radiative property is equivalent to the intrinsic radiative property 
only for the flat case $(\phi=\pi)$ and increases above the intrinsic value for all other cavity angles. The ability to achieve black behavior via the cavity effect is independent of material type, with all cavities approaching unity as the cavity angle collapses regardless of intrinsic radiative property. Lower intrinsic radiative properties experience rapid apparent property variation in the small angle range, whereas $\mathrm{V}$-grooves with intrinsic properties near unity experience the most rapid apparent property variation in the mid-angle range.

\subsubsection{Apparent Absorptivity for Collimated Irradiation}

Figs. $5 \mathrm{a}$ and $5 \mathrm{~b}$ illustrate the analytically-derived apparent absorptivity results for collimated irradiation at collimation angles of $\pi / 18\left(10^{\circ}\right)$ and $2 \pi / 9\left(40^{\circ}\right)$, respectively. The vertical dashed line in each plot indicates the separation point between full and partial illumination (where full illumination occurs to the right and partial illumination to the left). The analytical results were obtained with the use of the full illumination model as reported from Sparrow and Lin [12] and the partial illumination model developed in this work.

Unlike the case for diffuse irradiation, the apparent absorptivity for a specular V-groove exposed to collimated irradiation does not begin to increase immediately as the cavity angle decreases. For a collimated irradiation angle of $\gamma=\pi / 18$, the apparent absorptivity remains equivalent to the intrinsic absorptivity from approximately $\phi=\pi$ to $2 \pi / 3$ regardless of intrinsic absorptivity. This behavior is due to the specular nature of the surface, causing all rays to be reflected away from the cavity when the surface is mostly flat ( $\phi=\pi$ to $2 \pi / 3$ ). At around $\phi=2 \pi / 3$ the rays reflected after initial contact with one $\mathrm{V}$-groove surface begin to make contact with the opposite surface and the apparent absorptivity experiences a discontinuity in slope, suddenly increasing towards unity as the cavity angle continues to collapse towards zero. In general, the presence of collimated irradiation results in discontinuous behavior due to the similar reflection patterns experienced by all of the incident collimated rays.

An interesting behavior is encountered for larger collimation angles (Fig. 5b), where the apparent absorptivity decreases slightly with decreasing cavity angle over the range of approximately $\phi=5 \pi / 6$ to $\pi / 2$, as shown in Figure $5 b$. In this cavity angle range, for $\gamma=2 \pi / 9$, the rays initially striking the left surface reflect one time before exiting the cavity whereas the rays that strike the right surface reflect twice. As the cavity angle decreases, the percentage of rays 
falling on the right side of the surface decreases while the percentage of rays falling on the left surface increases, causing the total number of reflections experienced by all rays in the cavity to decrease. This slight decrease in apparent absorptivity as the cavity angle decreases disqualifies the intuitive assumption that a deeper cavity is always a more effective absorber for the case of specular reflection and collimated irradiation.

\section{CONCLUSIONS}

Existing models to calculate the apparent absorptivity of a diffusely-irradiated V-groove [28] (equivalent to the apparent emissivity of an isothermal V-groove), and the apparent absorptivity of a fully illuminated cavity subject to collimated irradiation [12] were verified against ray tracing results with an average discrepancy of less than $4.9 \times 10^{-4}$. A new analytical model was developed to calculate the apparent absorptivity for a partially illuminated, infinite $\mathrm{V}$ groove subject to collimated irradiation. This model has also been verified with ray tracing results with an average discrepancy of $4.6 \times 10^{-4}$. Results show that significant control of apparent radiative properties is possible by controlling the V-groove cavity angle, confirming the use of origami tessellations as possible variable emissivity surfaces.

\section{ACKNOWLEDGEMENTS}

This work was funded by a NASA Space Technology Research Fellowship, grant number NNX15AP49H 


\section{APPENDIX}

A ray at a given point of origin $\left(x_{1}, y_{1}\right)$ and ray-vertical angle $\chi_{1}$, measured clockwise from the vertical axis (Fig. 2b), intersects the left surface of the $\mathrm{V}$-groove at the location $X^{\prime}$ '. The rayvertical angle $\chi_{1}$ is equivalent to the collimation angle $\gamma$ and the point of origin is the right-most point of the cavity opening, where the coordinate system origin is located at the V-groove apex (Fig. 2b). The ray is described mathematically as a line using Eq. 15, where $x_{i}$ and $y_{i}$ represent a known point on the ray line (in this case $x_{1}$ and $y_{1}$ ), and $\chi_{i}$ is the ray-vertical angle of the ray (in this case $\gamma$ ). The left and right sides of the $\mathrm{V}$-groove are also defined mathematically as lines (see Eqs. 16 and 17, respectively).

In order to determine the intersection point of the ray and a cavity wall, the $x$ intersection point of the line defining the ray (Eq. 15) and the line defining the V-groove side that the ray is travelling towards (Eq. 16 or 17) is determined by equating the $y$ terms of Eq. 15 and Eq. 16 for a left-side intersection point or Eq. 15 and Eq. 17 for a right-side intersection point. A ray collides with a V-groove side of length $L$ (Fig. 1a) if an $x$ intersection value falls within the range $[-L \sin (\phi / 2)$ , 0] for a left-side intersection or $[0, L \sin (\phi / 2)]$ for a right-side intersection. If an intersection occurs, the ray is reflected specularly as shown in Fig. 6, using Eq. 18 for a left-side intersection or Eq. 19 for a right side intersection to determine the ray-vertical angle of the reflected ray $\left(\chi_{i+1}\right)$.

$$
\begin{gathered}
y=\frac{1}{\tan \left(\chi_{i}\right)} x+y_{i}-\frac{1}{\tan \left(\chi_{i}\right)} x_{i} \\
y=\frac{-x}{\tan \left(\frac{\phi}{2}\right)} \text { left side } \\
y=\frac{x}{\tan \left(\frac{\phi}{2}\right)} \text { right side } \\
\chi_{i+1}=\chi_{i}+\left[\pi-2\left(\chi_{i}+\frac{\phi}{2}\right)\right] \text { left intersection } \\
\chi_{i+1}=\chi_{i}+\left[\pi-2\left(\chi_{i}-\frac{\phi}{2}\right)\right] \text { right intersection }
\end{gathered}
$$


Once a ray has been reflected, a new line is calculated defining the direction of the reflected ray (Eq. 15), where $x_{i}$ and $y_{i}$ are now the latest intersection point of the ray $\left(x_{2}\right.$ and $y_{2}$ as shown in Fig. $2 \mathrm{~b}$ ) and $\chi_{i}$ is the new ray-vertical angle ( $\chi_{2}$ in Fig. 2b). Once again, the intersection point is calculated and a new ray line is calculated if a reflection occurs. This pattern continues until the ray leaves the cavity as determined by the $x$ intersection values. The number of reflections that the ray experienced is then counted and assigned to the value $n$. 


\section{NOMENCLATURE}

$m$

n

$N$

$N_{T}$

$N_{a}$

$q_{a}$

$q_{t}$

$\vec{r}_{1}$

$\vec{r}_{2}$

SE

$\vec{v}$

$x_{i}$

$X_{n}$

$X^{\prime}$

$X^{\prime \prime}$

$y_{i}$
Maximum number of reflections experienced by rays that impact the right surface

Maximum number of reflections experienced by rays that impact the left surface

Number of tests used in determining the standard error of the mean

Total number of rays emitted in a ray tracing test

Total number of rays absorbed by cavity surfaces

Amount of energy absorbed by the cavity surface [W]

Total amount of energy incident on the cavity opening [W]

Incoming ray vector

Outgoing ray vector

Standard deviation of the mean for 20 ray tracing tests

Standard error of the mean for $N$ completed ray tracing tests

Surface normal used in calculating reflected ray

$\mathrm{X}$ location of the $\mathrm{i}^{\text {th }}$ impact of the ray used in the simple ray tracing routine $[\mathrm{m}]$

Fraction of rays incident on the left panel that will reflect $n$ times

Normalized length from the bottom of the V-groove along the left panel to the initial intersection of a ray that grazes the top the cavity when exiting

Normalized length of the shadowed portion for the left panel

Y location of the $\mathrm{i}^{\text {th }}$ impact of the ray used in the simple ray tracing routine $[\mathrm{m}]$ 


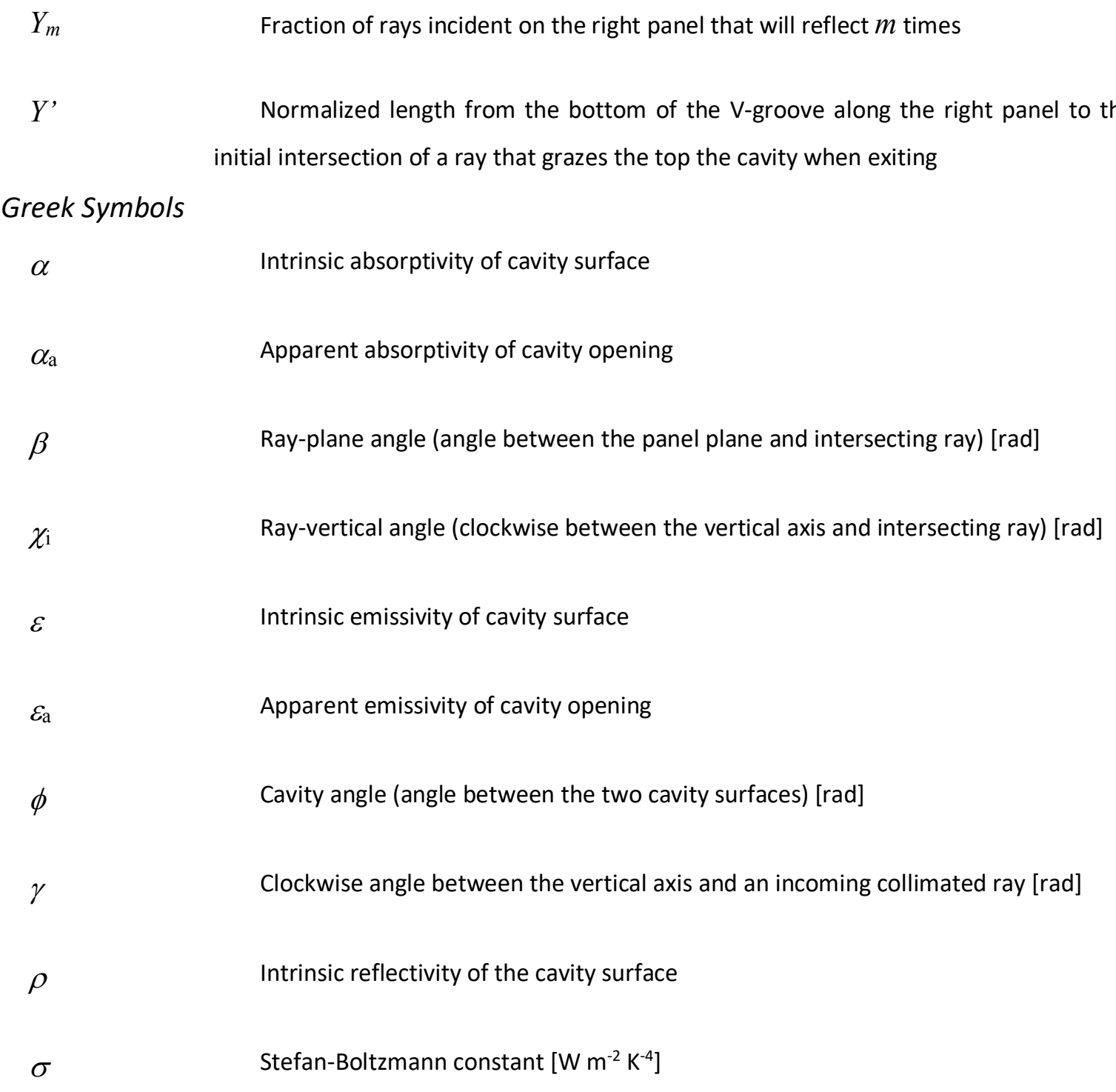

\section{REFERENCES}

[1] Miura, K., 1985, "Method of packaging and deployment of large membrances in space," The Institute of Space and Astronautical Science, 618, pp. 1-9.

[2] Evans, T. A., Lang, R. J., Magleby, S. P., and Howell, L. L., 2015, "Rigidly foldable origami gadgets and tessellations," R Soc Open Sci, 2(9), p. 150067.

[3] Karim, M. A., and Hawlader, M. N. A., 2006, "Performance investigation of flat plate, vcorrugated and finned air collectors," Energy, 31(4), pp. 452-470.

[4] Kabeel, A. E., Khalil, A., Shalaby, S. M., and Zayed, M. E., 2016, "Experimental investigation of thermal performance of flat and v-corrugated plate solar air heaters with and without pcm as thermal energy storage," Energy Conv. Manag., 113, pp. 264-272. 
[5] El-Sebaii, A. A., Aboul-Enein, S., Ramadan, M. R. I., Shalaby, S. M., and Moharram, B. M., 2011, "Investigation of thermal performance of-double pass-flat and v-corrugated plate solar air heaters," Energy, 36(2), pp. 1076-1086.

[6] Bailey, S. G., Fatemi, N., Landis, G. A., Brinker, D., and Faur, M., April 23 - 25, 1990, "Application of $v$ groove technology to InP solar cells," Proc. Indium Phosphide and Related Materials, Second International Conferece, IEEE, Denver, CO. April 23 - 25, 1990.

[7] Hollands, K. G. T., 1963, "Directional selectivity, emittance, and absorptance properties of vee corrugated specular surfaces," Solar Energy, 7(3), pp. 108 - 116.

[8] Mulford, R. B., Collins, N. S., Farnsworth, M. S., Jones, M. R., and Iverson, B. D., 2018, "Total hemispherical apparent radiative properties of the infinite $V$-groove with diffuse reflection," Under Review.

[9] Bedford, R. E., 1997, "Blackbodies as absolute radiation standards," Advances in Geophysics, 14, pp. 165-202.

[10] Prokhorov, A. V., Hanssen, L. M., and Mekhontsev, S. N., 2009, "Calculation of the radiation characteristics of blackbody radiation sources," Radiometric Temperature Measurements: I. Fundamentals, pp. 181-240.

[11] Steinfeld, A., 1990, "Apparent absorptance for diffusely and specularly reflecting spherical cavities," International Journal of Heat and Mass Transfer, 34(7), pp. 1895-1897.

[12] Sparrow, E. M., and Lin, S. H., 1962, "Absorption of thermal radiation in a v-groove cavity," International Journal of Heat and Mass Transfer, 5, pp. 1111-1115.

[13] Bedford, R. E., 1972, "Effective emissivities of blackbody cavities - a review," Temperature, It's Measurement and Control in Science and Industry, 4(1), pp. 425-434.

[14] Safwatt, H. H., 1970, "Absorption of thermal radiation in a hemispherical cavity," Journal of Heat Transfer, 92(1), pp. 198-201.

[15] De Lucas, J., 2015, "A simple geometrical model for calculation of the effective emissivity in blackbody cylindrical cavities," International Journal of Thermophysics, 36(2-3), pp. 267282.

[16] Ohwada, Y., 1988, "Mathematical proof of an extended kirchhoff law for a cavity having direction-dependent characteristics," J. Opt. Soc. Am. A-Opt. Image Sci. Vis., 5(1), pp. 141145.

[17] Kowsary, F., and Mahan, J. R., 2006, "Radiative characteristic of spherical cavities with specular reflectivity component," Journal of Heat Transfer, 128(3), p. 261.

[18] Alfano, G., 1972, "Apparent thermal emittance of cylindrical enclosures with and without diaphragms," International Journal of Heat and Mass Transfer, 15(12), pp. 2671-2674.

[19] Buckley, H., 1927, "On the radiation from the inside of a circular cylinder," Philosophical Magazine, 4, pp. 753-762.

[20] Alfano, G., and Sarno, A., 1975, "Normal and hemispherical thermal emittances of cylindrical cavities," Journal of Heat Transfer, 97(3), pp. 387-390.

[21] Bedford, R. E., and Ma, C. K., 1974, "Emissivities of diffuse cavities isothermal and nonisothermal cones and cylinders," Journal of the Optical Society of America, 64(3), pp. 339-349. 
[22] Zipin, R. B., 1966, "The apparent thermal radiation properties of an isothermal v-groove with specularly reflecting walls," Journal of Research of the National Bureau of Standards, 70c(4), pp. 275-280.

[23] Mulford, R. B., Jones, M. R., and Iverson, B. D., 2015, "Dynamic control of radiative surface properties with origami-inspired design," Journal of Heat Transfer, 138(3), p. 032701.

[24] Sparrow, E. M., and Eckert, E. R. G., 1961, "Radiative heat exchange between surfaces with specular reflection," International Journal of Heat and Mass Transfer, 3, pp. 42-54.

[25] Sparrow, E. M., Eckert, E. R. G., and Jonsson, V. K., 1962, "An enclosure theory for radiative exchange between specularly and diffusely reflecting surfaces," Journal of Heat Transfer, 84(4), pp. 294-299.

[26] Zipin, R. B., 1965, "The directional spectral reflectance of well characterized symmetric v grooved surfaces," Doctorate, Purdue University, Ann Arbor, Michigan.

[27] Daws, L. F., 1954, "The emissivity of a groove," British Journal of Applied Physics, 5, pp. 182 $-187$.

[28] Modest, M. F., 2013, Radiative Heat Transfer, Academic Press, New York, NY.

[29] Le Hardy, D., Favennec, Y., and Rousseau, B., 2016, "Solution of the 2-D steady-state radiative transfer equation in participating media with specular reflections using SUPG and DG finite elements," Journal of Quantitative Spectroscopy and Radiative Transfer, 179, pp. 149-164.

[30] Le Hardy, D., Favennec, Y., Rousseau, B., and Hecht, F., 2017, "Specular reflection treatment for the 3D radiative transfer equation solved with the discrete ordinates method, "Journal of Computational Physics, 334, pp. 541-572.

[31] Sapritsky, V. I., and Prokhorov, A. V., 1992, "Calculation of the effective emissivities of specular diffuse cavities by the monte carlo method," Metrologia, 29(1), pp. 9-14.

[32] Prokhorov, A. V., 1998, "Monte carlo method in optical radiometry," Metrologia, 35(4), pp. 465-471.

[33] Baumeister, J. F., 1990, "Thermal radiation characteristics of nonisothermal cylindrical enclosures using a numerical ray tracing technique," Proc. 5th Thermophysics and Heat Transfer Conference, AIAA and ASME, Seattle, WA. June 18 - 20, 1990.

[34] Baumeister, J. F., 1993, "Application of ray tracing in radiation heat transfer," Proc. Thermal and Fluids Analysis Workshop, NASA, Cleveland, OH, pp. 1-53. August 16 - 20, 1993.

[35] Foley, J., van Dam, A., Feiner, S., and Hughes, J., 2013, "Clipping lines," Computer Graphics, Addison-Wesley Profesional, Boston, MA.

[36] Navidi, W., 2010, Statistics for Engineers and Scientists, McGraw-Hill, New York, NY. 


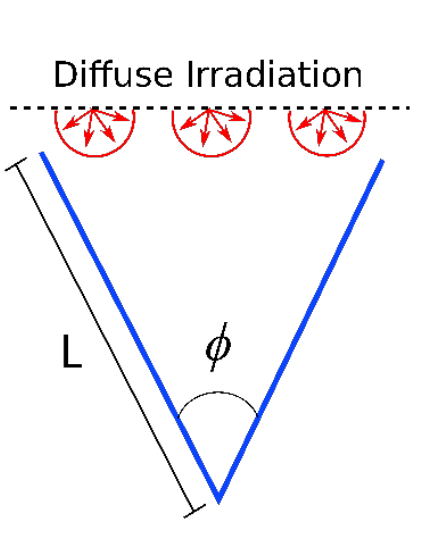

(a)
Collimated Irradiation

Full Illumination $(\gamma \leq \phi / 2)$

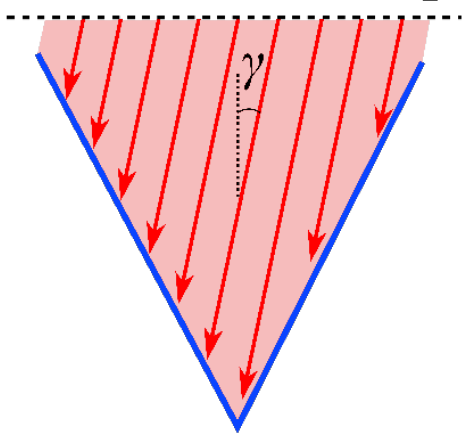

(b)
Collimated Irradiation

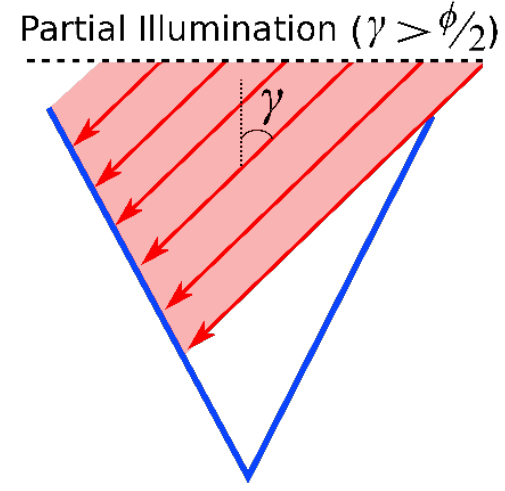

(c)

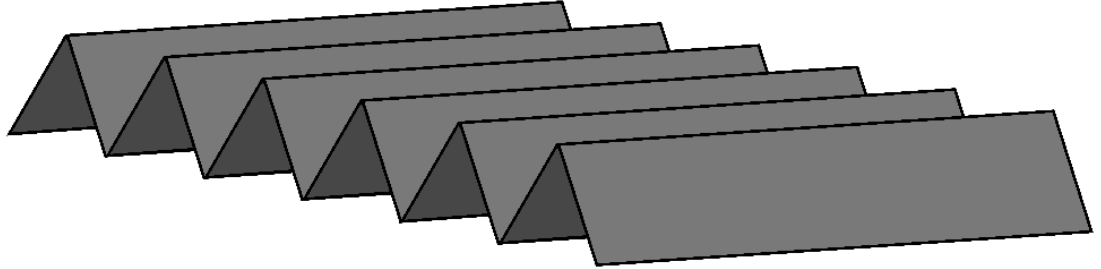

(d)

Figure 1 - (a) An infinite V-groove with diffuse irradiation. Cavity dimensions are governed by sides of length $L$ and an included angle of $\phi$. (b) An infinite $V$-groove with collimated irradiation and full illumination where the irradiation falls initially on both sides of the V-groove; the required condition for $\gamma$ and $\phi$ is shown in the figure. (c) An infinite $V$-groove with collimated irradiation and partial illumination where the irradiation falls only on one side of the V-groove; the required condition for $\gamma$ and $\phi$ is shown in the figure. (d) A 3D depiction of an accordion tessellation. 


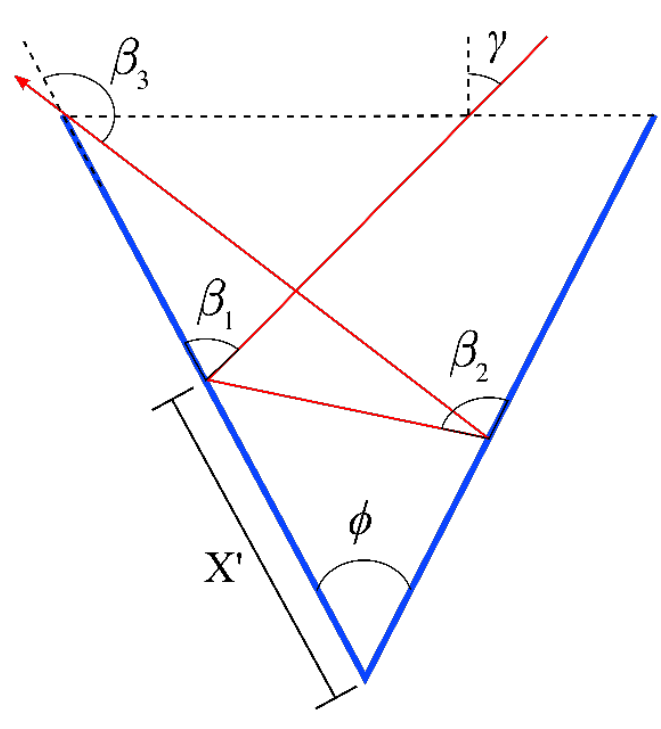

(a)

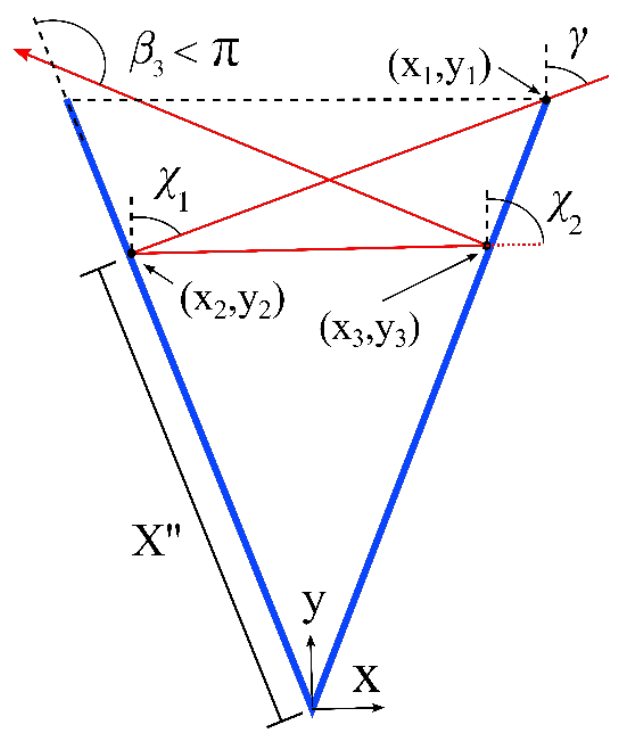

(b)

Figure 2 - (a) Definition of ray-plane angle $(\beta)$ and $X^{\prime}$. A ray at an angle of $\gamma$ enters a $\mathrm{V}$ groove cavity and intersects the left cavity wall at $X$. The ray is reflected from the cavity walls twice before exiting the cavity through the left-most point of the cavity opening. Any ray that enters the cavity opening further to the right of this ray, striking below the point $X^{\prime}$, will reflect one additional time before reflecting out of the cavity opening. All rays that enter to the left of the initial ray, striking above the point $X^{\prime}$, will reflect the same number of times as the indicated ray. The ray-plane angle $\beta$ is depicted for both of the internal reflections and at the exit. (b) Definition of ray-vertical angle $(\chi)$ and $X$ '. A ray enters the right-most point of the cavity opening with an initial ray-vertical angle $\left(\chi_{1}\right)$ equivalent to the ray's collimation angle $(\gamma)$. The ray strikes the left wall at the point $X$ ' which represents the fraction of the left wall that is irradiated since no rays will intersect below this point initially. After an additional reflection, the ray exits the cavity opening. The ray-plane angle between the theoretical extension of the cavity wall and the exiting ray $\left(\beta_{3}\right)$ does not exceed $\pi$, violating the condition used in the full illumination case used to determine when a ray has left the cavity. 


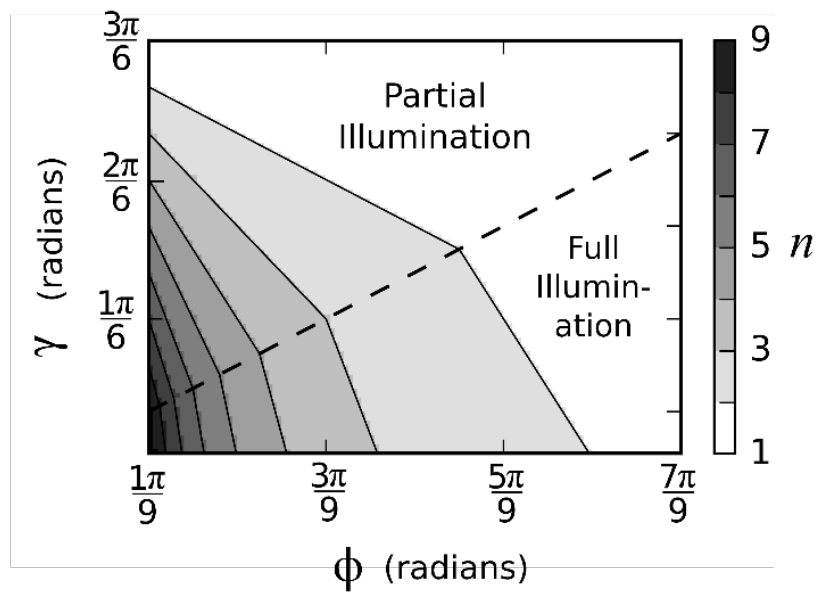

Figure 3 - Maximum number of reflections $(n)$ encountered by a ray entering a V-groove cavity from the right-most location of the cavity opening as a function of ray inclination angle $(\gamma)$ and V-groove cavity angle $(\phi)$, determined using the $2 \mathrm{D}$ ray tracing routine described in the Appendix. The dashed line depicts the relationship $\gamma=\phi / 2$ and separates the fully and partially illuminated regions as indicated on the image. Distinct linear patterns for the number of reflections are visible and have been marked with solid black lines. Data was calculated over the full range of collimation angles $(0<\gamma<\pi / 2)$ and cavity angles $(0<\phi<\pi)$ in increments of $\pi / 180$. However, the data is displayed over a limited $\phi$ range to better illustrate the results. 


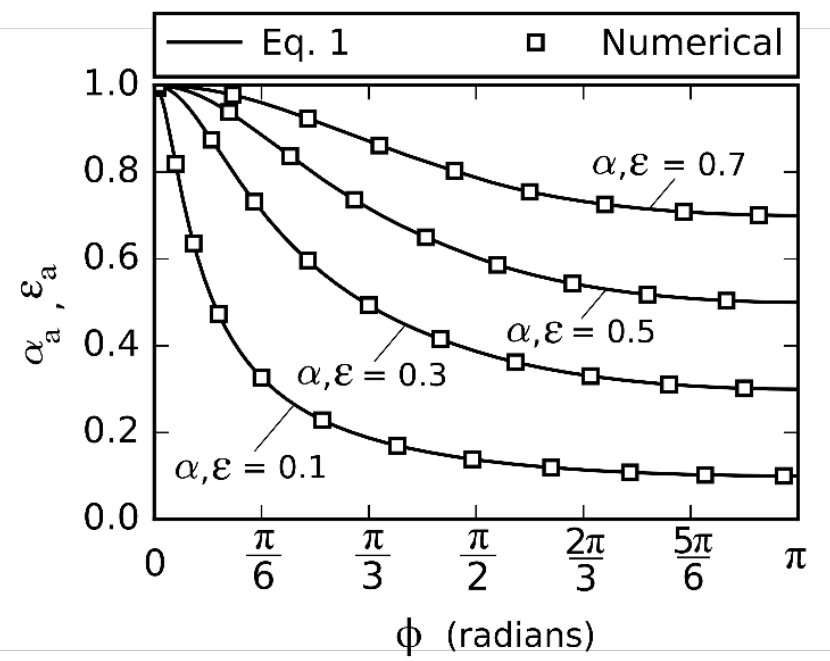

Figure 4 - The apparent absorptivity of a specularly-reflecting infinite V-groove subject to diffuse irradiation as a function of cavity angle $(\phi)$ and intrinsic surface absorptivity $(\alpha)$. Results in this plot also apply to the apparent emissivity of an isothermal infinite V-groove as a function of cavity angle and intrinsic surface emissivity [16]. Agreement between ray tracing results and analytical results has an average discrepancy of $4.4 \times 10^{-4}$. 


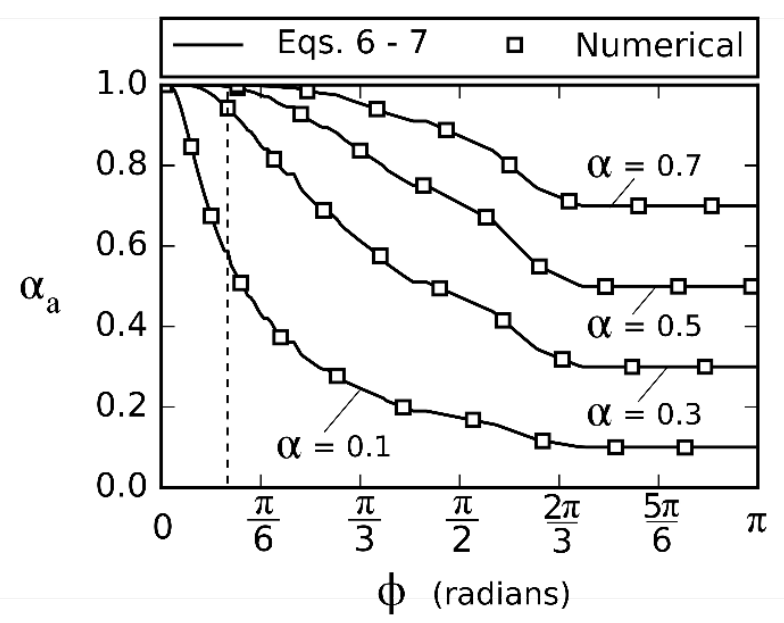

(a)

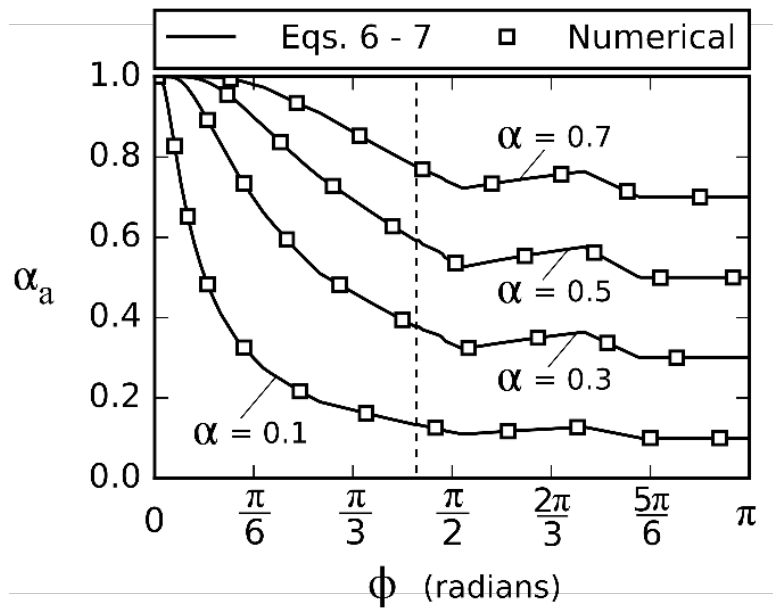

(b)

Figure 5 - (a) Apparent absorptivity of a specularly-reflecting infinite $V$-groove subject to collimated irradiation with an inclination angle of $\gamma=\pi / 18\left(10^{\circ}\right)$ as a function of cavity angle $(\phi)$ and intrinsic surface absorptivity $(\alpha)$. Solid lines were calculated by Eq. 6 for full illumination, $\phi \geq \pi / 9\left(20^{\circ}\right)$, and Eq. 7 for partial illumination, $\phi<\pi / 9\left(20^{\circ}\right)$. Data indicated by the squares were obtained by Monte Carlo ray tracing; only a portion of the numerical data points are depicted here for clarity. Agreement between the analytical and numerical methods is excellent with an average discrepancy of $4.9 \times 10^{-4}$ to the right of the dotted line and $4.6 \times 10^{-4}$ to the left of the dotted line. (b) Apparent absorptivity of a specularly-reflecting infinite V-groove subject to collimated irradiation with an inclination angle of $\gamma=2 \pi / 9\left(40^{\circ}\right)$ as a function of cavity angle and intrinsic surface absorptivity. Partial illumination occurs at cavity angles less than $4 \pi / 9\left(80^{\circ}\right)$ as indicated by the vertical dashed line. Agreement between the analytical model and numerical data is the same as in Fig. 4a. A slight increase in the apparent absorptivity is visible between the cavity angles of $5 \pi / 6\left(150^{\circ}\right)$ and $\pi / 2\left(90^{\circ}\right)$, indicating that apparent radiative properties do not always increase as the cavity angles decreases. 

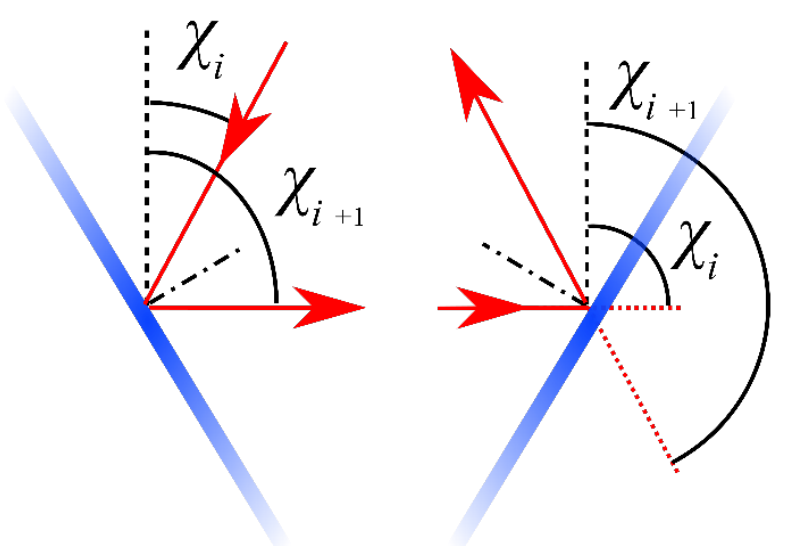

Figure 6 - The ray-vertical angles of a specularly-reflected ray before $\left(\chi_{i}\right)$ and after $\left(\chi_{i+1}\right)$ experiencing a reflection from the left or right surfaces of a V-groove cavity. The reflected ray angle is determined using Eqs. 18 or 19 for the left and right cavity surfaces, respectively. 


\begin{tabular}{|c|c|c|c|c|c|}
\hline $\begin{array}{c}\text { Desired } \\
\text { Output }\end{array}$ & \multicolumn{2}{|c|}{ Conditions } & $\begin{array}{c}\text { Required } \\
\text { Inputs }\end{array}$ & $\begin{array}{l}\text { Equation } \\
\text { Numbers }\end{array}$ & Source \\
\hline \multirow{2}{*}{$\varepsilon_{a}$} & Emission: & Isothermal, Diffuse & \multirow{2}{*}{$\varepsilon, \phi$} & \multirow{2}{*}{1} & \multirow{2}{*}[28]{} \\
\hline & Reflection: & Specular & & & \\
\hline \multirow{2}{*}{$\alpha_{a}$} & Irradiation: & Diffuse & \multirow{2}{*}{$\alpha, \phi$} & \multirow{2}{*}{1} & \multirow{2}{*}[28]{} \\
\hline & Reflection: & Specular & & & \\
\hline \multirow[t]{2}{*}{$\alpha_{a}$} & Irradiation: & $\begin{array}{l}\text { Collimated, } \\
\text { Fully illuminated } \\
(\gamma \leq \phi / 2)\end{array}$ & \multirow[t]{2}{*}{$\alpha, \phi, \gamma$} & \multirow[t]{2}{*}{3,4 and 6} & \multirow[t]{2}{*}[12]{} \\
\hline & Reflection: & Specular & & & \\
\hline \multirow[t]{2}{*}{$\alpha_{a}$} & Irradiation: & $\begin{array}{l}\text { Collimated, } \\
\text { Partially illuminated } \\
(\gamma>\phi / 2)\end{array}$ & \multirow[t]{2}{*}{$\alpha, \phi, \gamma$} & \multirow[t]{2}{*}{$3,7-10$} & \multirow[t]{2}{*}{ This worl } \\
\hline & Reflection: & Specular & & & \\
\hline
\end{tabular}

Table 1 - Summary of the models for apparent radiative behavior summarized or developed in this work. The conditions required for the use of each model are listed as well as the desired apparent property. Equation numbers for calculation of the desired output are listed. 